\title{
Search for Nucleon Decays in Super-Kamiokande
}

\author{
Makoto Miura*† \\ ICRR \\ E-mail: miurameicrr.u-tokyo.ac.jp
}

Searches for nucleon decays are performed by Super-Kamiokande, a large water cherenkov detector. The total exposure is $173 \mathrm{kton} \cdot y e a r$ which includes 1489 days data of Super-Kamiokande phase I (SK-1), 799 days of phase II (SK-2), and 518 days of phase III (SK-3). We have not observed any evidence of nucleon decay yet. The lower limit of proton life time decay into $e^{+} \pi^{0}$, which is dominant mode predicted by non-SUSY model, is $1.0 \times 10^{34}$ years with $90 \%$ confidence level. The lower limit of proton life time decay into $\bar{v} K^{+}$, which is dominant mode predicted by SUSY model, is $3.3 \times 10^{33}$ years with $90 \%$ confidence level.

35th International Conference of High Energy Physics

July 22-28, 2010

Paris, France

* Speaker.

${ }^{\dagger}$ A footnote may follow. 


\section{Introduction}

In Grand Unified Theory (GUTs), nucleon decay can proceed via an exchange of a massive boson between two quarks in a nucleon, and one quark transforms into a lepton and another into an anti-quark which binds a spectator quark creating a meson. The favored decay mode in GUTs based on SU(5) symmetry is $p \rightarrow e^{+} \pi^{0}$. On the other hand, GUTs model incorporating supersymmetry (SUSY-GUTs) suppress the decay mode $p \rightarrow e^{+} \pi^{0}$ but favor the other mode $p \rightarrow \bar{v} K^{+}$ via dimension five operator interactions with the exchange of a heavy supersymmetric color triplet Higgsino.

The Super-Kamiokande is a large water cherenkov detector with 22.5 kton fiducial volume, $7 \times 10^{33}$ protons. A detailed description of the Super-Kamiokande detector can be found else where [1]. The Super-Kamiokande started observation from April 1996 with 11,146 PMTs in inner detector (ID) which covered $40 \%$ of ID surface. The observation was continued until July 2001, corresponding to 1489.2 days, $91.7 \mathrm{kton} \cdot \mathrm{year}$, and this period is called Super-Kamiokande phase I (SK-1). After an accident in 2001, about half of ID PMTs were lost and the detector was reconstructed with 5,182 ID PMTs, decreasing photo coverage to $19 \%$. The period from December 2002 until October 2005, corresponding to 798.6 days, $49.2 \mathrm{kton} \cdot$ year, is called phase II (SK-2). After full reconstruction in 2006, the photo coverage has been recovered up to $40 \%$, and the period until electronics update in 2008 is called phase III (SK-3), with 518.1 days, 31.9 kton-year.

The results of $p \rightarrow e^{+} \pi^{0}$ with SK1 and SK2 data, and $p \rightarrow \bar{v} K^{+}$for SK-1 data were already published [目] [3]. In this paper, the results of combined analysis of SK-1 to SK-3 are reported.

\section{2. $p \rightarrow e^{+} \pi^{0}$}

If a proton decays into $e^{+}$and $\pi^{0}$, they are emitted back-to-back in the proton rest frame. The $\pi^{0}$ immediately decays into two gamma rays. In $p \rightarrow e^{+} \pi^{0}$ mode, the following selection criteria are applied: (A1) the vertex is in the fiducial volume, and the event is fully contained in the detector (FC event), (A2) the number of rings is two or three and all rings are e-like, (A3) there is no Michel electron, (A4) for three ring events, $\pi^{0}$ invariant mass is reconstructed in between 85 to 185 $\mathrm{MeV} / \mathrm{c}^{2}$, (A5) the reconstructed total momentum is less than $250 \mathrm{MeV} / \mathrm{c}$ and the reconstructed total invariant mass is in between 800 to $1050 \mathrm{MeV} / \mathrm{c}^{2}$. Figures 1 show total momentum versus total invariant mass after (A1)-(A4) cuts, for the proton decay MC, the atmospheric neutrino MC, and the data. The detection efficiencies are estimated to be 44.6, 43.5, and 45.2\% for SK-1, SK-2, and SK-3, respectively. The inefficiency is mainly due to nuclear interaction effects of pions in oxygen. The total systematic uncertainties are estimated to be $19 \%$ for all period and the largest contribution is the uncertainty of the cross section for pion-nuclear effects (15\%). Background for proton decay analysis is atmospheric neutrino interaction. It is estimated by the atmospheric neutrino MC with large statistics (500 years equivalent for each period). After all cuts, The remaining backgrounds are estimated to be $0.20,0.11$, and 0.06 events for SK-1, SK-2, and SK-3 period, respectively. The dominant background source is charged current (CC) single-pion production (32\%). The number of observed data in the signal box in Figure 11 are 0 for all period. The life time limit calculated by a method based on Bayes theorem is $\tau / B_{p \rightarrow e^{+} \pi^{0}}>1.0 \times 10^{34}$ years at $90 \%$ confidence level. 

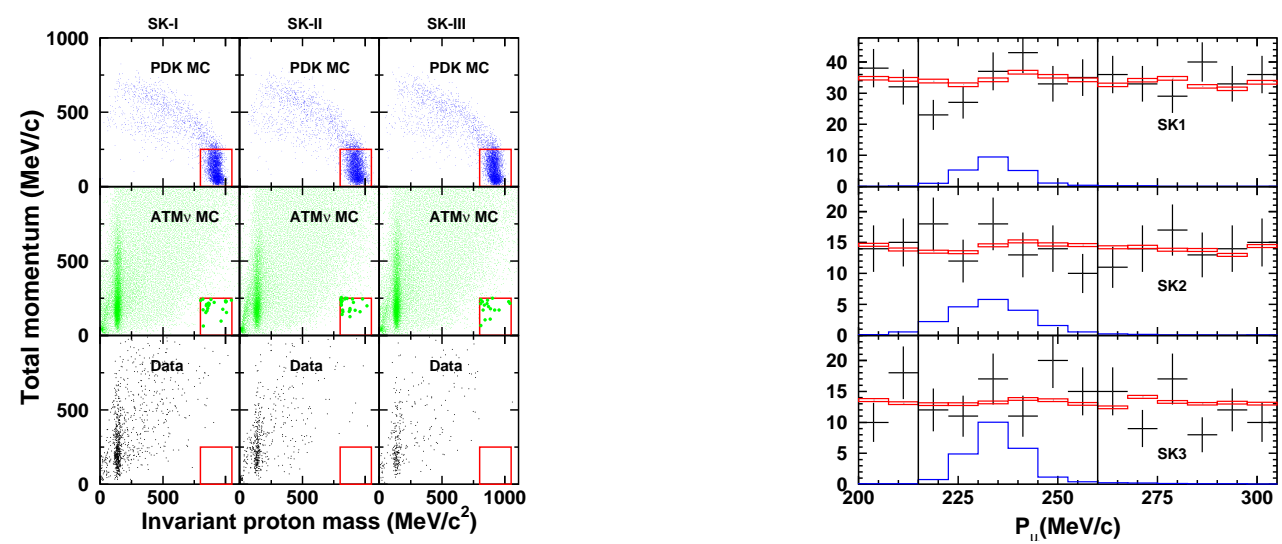

Figure 1: Horizontal axis is total mass and vertical Figure 2: Momentum distribution of FC1R $\mu$ axis is total momentum for proton decay MC (upper), like sample of SK-1 (top), SK-2(middle), and SKatmospheric MC (middle), and data (bottom), SK1, 3(bottom). Cross dots are data, boxes are atmospheric SK2, and SK3 from left. Red box is signal region. neutrino MC, and histograms are proton decay MC.

\section{3. $p \rightarrow \bar{v} K^{+}$}

If a proton decays into $K^{+}$and $\bar{v}$, the momentum of $K^{+}$is below cherenkov threshold and it cannot emit cherenkov light. Most of $K^{+}$are stopped in water and decay into other particles. For $p \rightarrow \bar{v} K^{+}$mode, three methods are employed to analyze and merged results are shown later section.

3.1 $K^{+} \rightarrow \mu^{+} v_{\mu}$

A $K^{+}$decays into $\mu^{+} v_{\mu}$ with $63.5 \%$ fraction and they have monochromatic momentum (236 $\mathrm{MeV} / \mathrm{c}$ ) because most of $\mathrm{K}^{+}$are stopped and decay. So we may see event excess around this momentum region if the protons decay in this mode. Selection criteria requires; (B1) FC event in the fiducial volume, (B2) single $\mu$-like ring, (B3) there is one Michel electron, (B0) events are not selected by the next method (see the next sub section). Then the momentum distribution of $\mu$ in data are divided into 3 regions; $200-215 \mathrm{MeV} / \mathrm{c}, 215-260 \mathrm{MeV} / \mathrm{c}$, and $260-300 \mathrm{MeV} / \mathrm{c}$ and compared by atmospheric neutrino MC and proton decay MC. Figures 2 show muon momentum distributions of data, atmospheric neutrino MC, and proton decay MC. The data and atmospheric neutrino MC agree well and there is no indication of the proton decay.

\section{2 $K^{+} \rightarrow \mu^{+} v_{\mu}$ with prompt $\gamma$}

There are much background events in single ring $\mu$ sample as shown in the previous section. To eliminate the background, further cuts are applied. If a proton decays in the oxygen, the remaining nitrogen nucleus left in excited states emit $\gamma$ rays immediately. The most significant branch is the $6.32 \mathrm{MeV} \gamma$ ray from $p_{3 / 2}$ hole state with $41 \%$ probability [ [ $₫$. To select $K^{+} \rightarrow \mu^{+} v_{\mu}$ with prompt $\gamma$, the following selection criteria are applied after (B1)-(B3) in section 3.1; (B4) the distance between $\mu$ and decay electron is less than $200 \mathrm{~cm}$, (B5) the goodness of vertex fit is greater than 0.6. Then a time window with $12 \mathrm{nsec}$ width is opened before muon peak and is slide to earlier time to 
find a maximum hit cluster which may be produced by $\gamma$ ray. $N_{\gamma}$ and $T_{\gamma}$ are defined as the maximum number of hit in the time window and the time of middle of the time window. (B7) $8<N_{\gamma}<60$ for SK-1 and SK3, and $4<N_{\gamma}<30$ for SK-2, (B8) $T_{\mu}-T_{\gamma}<75$ nsec. The detection efficiencies are estimated to be 7.2, 5.8, and $7.3 \%$ for SK-1, SK-2, and SK-3, respectively. The systematic uncertainties are estimated to be $22 \%$ for all period, which are dominated by the uncertainty of the $\gamma$ ray emission probability (20\%). The background events are estimated to be $0.16,0.08$, and 0.03 events for SK-1, SK-2, and SK-3 period, respectively. The most dominant background is that the neutrino interacts with nuclei and makes resonance $\mathrm{N}(1650)$ and it decays into $K^{+} \Lambda$. On the other hand, there are no candidates in all period.

\section{$3.3 K^{+} \rightarrow \pi^{0} \pi^{+}$}

The momentum of $\pi^{+}$is just above cherenkov threshold and its cherenkov ring cannot be observed clearly. As a result, two $\gamma_{\mathrm{s}}$ from a $\pi^{0}$ and PMT activities in the backward of $\pi^{0}$ direction, and a decay electron should be observed. The selection criteria is required; (C1) FC 2 rings and both are e-like, (C2) one decay electron, (C3) $85<M_{\pi^{0}}<185 \mathrm{MeV}$, (C4) $175<P_{\pi^{0}}<250 \mathrm{MeV} / \mathrm{c}$. Then a backward electron-equivalent energy, $E_{b k}$ and a residual energy, $E_{\text {res }}$ are estimated from PMT charges in $140^{\circ} \sim 180^{\circ}$ and $90^{\circ} \sim 140^{\circ}$ from the $\pi^{0}$ direction. (C5) $7<E_{b k}<17 \mathrm{MeV}$, (C6) $E_{\text {res }}<12 \mathrm{MeV}$. The detection efficiencies are estimated to be $6.5,5.3$, and $6.6 \%$ for SK-1, SK-2, and SK-3, respectively. The systematic uncertainties are $8.6 \%$ both for all period and the most dominant error comes from uncertainty of $\mathrm{N}-\pi$ cross section in water $(5.0 \%)$. The numbers of backgrounds are estimated to be $0.46,0.33$, and 0.14 , for SK-1, SK-2, and SK-3, respectively. The large contributions are single $\pi$ production in charged and neutral current interactions. There are no candidates in the data.

\subsection{Combined limit for $p \rightarrow \bar{v} K^{+}$}

The three methods are used to calculate lifetime limit for the proton decay mode $p \rightarrow \bar{v} K^{+}$ based on the Bayes theorem. Detail descriptions can be found in reference [3]. The obtained lower limit of the proton lifetime via $p \rightarrow \bar{v} K^{+}$is $3.3 \times 10^{33}$ years at $90 \%$ confidence level.

\section{Summary}

We have searched for nucleon decay via $p \rightarrow e^{+} \pi^{0}$, which is dominant mode in the non-SUSY GUTs, and $p \rightarrow \bar{v} K^{+}$, which is dominant mode in the SUSY-GUTs, from an exposure of 173 kton year. No significant excesses above background of the atmospheric neutrino interactions are observed. The lower limits of the partial nucleon lifetime at $90 \%$ confidence level are $1.0 \times 10^{34}$ years and $3.3 \times 10^{33}$ years, respectively.

\section{References}

[1] Y. Fukuda et al. Nucl. Instrum. Methods A 501, 418 (2003).

[2] H. Nishino et al. Phys. Rev. Lett. 102, 141801 (2009).

[3] K. Kobayashi et al. Phys. Rev. D 72, 052007 (2005).

[4] H. Ejiri Phys. Rev. C 48, 1442 (1993). 